\section{Commentary: "Hot" gene signatures for checkpoint response prediction: A very cool idea}

\author{
Harvey I. Pass, MD
}

Lung cancers are pathologically and genomically heterogeneous, and this heterogeneity governs how an individual patient may recur after an R0 resection or respond to systemic therapy. Moreover, lung cancers are influenced by the soil in which they form and progress, now termed the tumor microenvironment (TME), and this microenvironment promotes immune evasion by checkpoint inhibitory molecules including CTLA-4 and PD-L1. Antibody-mediated inactivation of these inhibitory molecules has produced durable responses in a subset of patients with advanced tumors, and checkpoint inhibitors represent the newest first- and second-line lung cancer therapies. But prediction of response using such "biomarkers" as PD-L1 tumor staining or tumor mutation burden remains an inexact science. Why? Perhaps we should reconsider novel prediction, and the first step should be to consolidate immune heterogeneity of lung cancer into more immunologically relevant "bins" that have "hot" or immunologically alterable TME or "cold" for which other modalities should be considered, a strategy used by Jang and colleagues. ${ }^{2}$

The take-home message in this landmark report is that transcriptomic analysis of lung cancer histology bolstered by firstclass computational biology can possibly discover signatures for these "bins," both in adenocarcinoma and squamous cell carcinoma, and potentially improve selection of candidates for checkpoint inhibition. They defined clusters of patients who had all the characteristics for responding to immunotherapy: the correct white cell phenotype in the TME, elevated checkpoint markers, and theoretical in silico functional prediction of immune activation. But the proof of a signature must come from a population where you actually know what happened! These investigators applied a signature from an

\footnotetext{
From the Department of Cardiothoracic Surgery, Division of General Thoracic Surgery NYU Langone Medical Center, New York, NY.

Disclosures: Author has nothing to disclose with regard to commercial support.

Received for publication Dec 7, 2019; revisions received Dec 7, 2019; accepted for publication Dec 10, 2019; available ahead of print Feb 6, 2020.

Address for reprints: Harvey I. Pass, MD, Department of Cardiothoracic Surgery,

General Thoracic Surgery, NYU Langone Medical Center, 530 First Ave, 9V,

New York, NY 10016 (E-mail: Harvey.Pass@nyumc.org).

J Thorac Cardiovasc Surg 2020;159:1611-2

$0022-5223 / \$ 36.00$

Copyright (c) 2020 by The American Association for Thoracic Surgery

https://doi.org/10.1016/j.jtcvs.2019.12.043
}

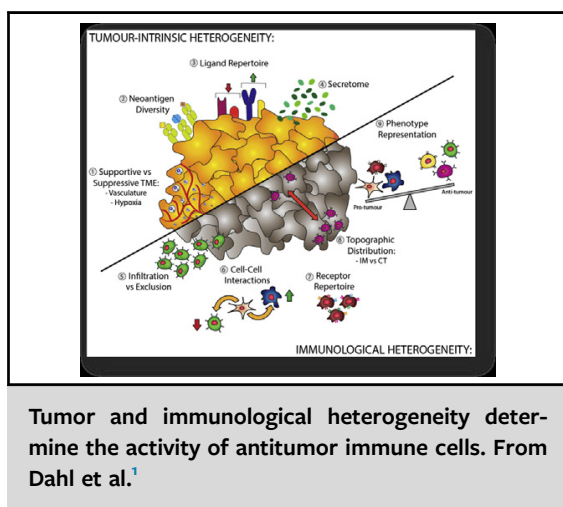

CENTRAL MESSAGE

Transcriptomic signatures can reduce lung cancer tumor microenvironment heterogeneity and potentially improve the sensitivity and specificity of immunotherapy response prediction.

immuno-treated population with known endpoints and reported that the "response" signature had high sensitivity and specificity for recognition of the "hot bins" of patients with lung cancer who should respond.

These findings are more than "proof of principle," and whether the "Barcelona" signature has use in prospective studies is secondary to the meaning of this paper. We need to apply and advance the ideas of this paper into as many prospective neoadjuvant immunotherapy or latestage immunotherapy trials. We already recognize that transcriptomics of fresh tissue using single-cell RNAseq may not only be able to define these types of signatures on an individual basis but also be able to validate in shortterm spheroid cultures whether these signatures are specific for a given therapy to be used. Combining such in vitro predictive assays with digital spatial profiling multiplex immunofluorescent platforms will allow us to then investigate the TME as different regions of immune cell/tumor interactions on a single slide. Specificity of individual treatment for a response is the holy grail of immunotherapy, considering the complexity and number of checkpoint molecules, and even after this is "standardized," we will need to further dial up sensitivity and specificity with neoantigen analyses.

There is much more to come, thoracic surgeons. This is a great paper to read slowly, understand the thinking behind it, and recognize it as a foundational piece of information when conversing with our medical colleagues. 


\section{References}

1. Dahl O, Dale JE, Brydøy M. Rationale for combination of radiation therapy and immune checkpoint blockers to improve cancer treatment. Acta Oncol. 2019;58: $9-20$.
2. Jang H, Lee H-S, Ramos D, Park IK, Kang CH, Burt VM, et al. Transcriptome-based molecular subtyping of non-small cell lung cancer may predict response to immune checkpoint inhibitors. J Thorac Cardiovasc Surg. 2020;159:1598-610.e3
See Article page 1598.

\section{Commentary: Breaking down non-small cell lung cancer tumor microenvironment heterogeneity and predicting response to immune checkpoint inhibitors}

\author{
Joshua Boys, MD, and Mark Onaitis, MD
}

Immune checkpoint inhibitors have brought new hope for improving survival in patients with non-small cell lung cancer (NSCLC). However, determinants of response to immune checkpoint therapy are unclear. Studies have shown a correlation of PDL-1 expression and response to checkpoint inhibitors, ${ }^{1,2}$ but many of us have seen patients respond to checkpoint inhibitors when their expression levels were not significant and vice versa, indicating that responses to checkpoint inhibitors are not always as simple as expression levels. In the current study, Jang and collealgues ${ }^{3}$ address the complexity of lung cancer microenvironments by using transcriptome-based molecular subtyping of NSCLC to predict response to immune checkpoint inhibitors. They used NSCLC mRNA transcriptomes in conjunction with tumor and immune cell function microenvironments to assess treatment response to anti-PD-1 therapy in 87 adenocarcinomas and 101 squamous cell carcinomas. Using mRNA transcriptomes for both adenocarcinoma and squamous cell carcinoma as the base subtypes, they additionally analyzed tumor and immune cell functional activity to classify the tumor

\footnotetext{
From the Division of Cardiothoracic Surgery, Department of Surgery, University of California San Diego, San Diego, Calif.

Disclosures: Authors have nothing to disclose with regard to commercial support.

Received for publication Nov 28, 2019; accepted for publication Nov 29, 2019; available ahead of print Feb 12, 2020.

Address for reprints: Mark Onaitis, MD, 9300 Campus Point Dr \#7892, La Jolla, CA 92037 (E-mail: monaitis@health.ucsd.edu).

J Thorac Cardiovasc Surg 2020;159:1612-3 0022-5223/ $\$ 36.00$

Copyright $\Subset 2019$ Published by Elsevier Inc. on behalf of The American Association for Thoracic Surgery

https://doi.org/10.1016/j.jtcvs.2019.11.097
}

Check for updates

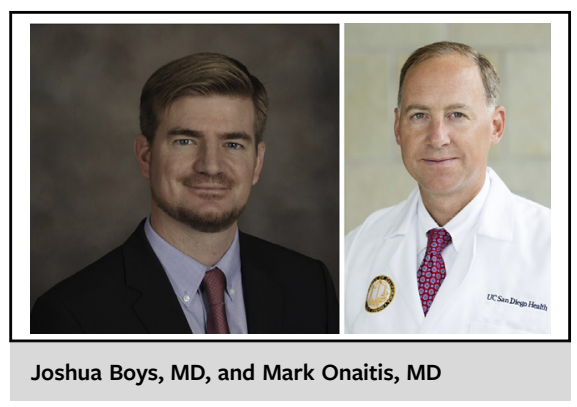

CENTRAL MESSAGE

The study in the current issue sheds new light on how the microenvironment of tumors may predict response to immune modulators.

microenvironment as "good" or "bad" for response to therapy. They then used previously published mRNA data from a Barcelona cohort of 35 patients with NSCLC treated with anti-PD-1 therapy to perform forward and backward modeling for response to treatment. ${ }^{4}$ In both the forward and backward models they found a high concordance with treatment response in the "good" subtype for both adenocarcinoma and squamous cell carcinoma.

Although it is limited by small sample size and does not consider the gold standard of PD1 or PD-L1 protein expression by immunohistochemistry nor tumor mutation burden, this is an interesting study in that it identifies subgroups of patients that seem to benefit from immune checkpoint therapy. The authors should be congratulated for trying to measure the complexity of tumor microenvironment and how it potentially affects response to anti-PD-1 therapy. More and more often, tumors are sequenced for evaluation of therapeutic targets. This sort of analysis may allow combination of genetic targets and immune checkpoint inhibitors. 